\title{
Nuclear effects in Drell-Yan production at the LHC
}

\author{
M. Krelina ${ }^{1, a}$, E. Basso ${ }^{2,3}$, V. P. Goncalves ${ }^{3,4}$, J. Nemchik ${ }^{1,5}$, and R. Pasechnik ${ }^{3}$ \\ ${ }^{1}$ FNSPE, Czech Technical University in Prague, Brehova 7, 11519 Prague , Czech Republic \\ ${ }^{2}$ Instituto de FIsica, Universidade Federal do Rio de Janeiro, Caixa Postal 68528, Rio de Janeiro, RJ 21941- \\ 972, Brazil \\ ${ }^{3}$ Department of Astronomy and Theoretical Physics, Lund University, SE-223 62 Lund, Sweden \\ ${ }^{4}$ High and Medium Energy Group, Instituto de Fisica e Matematica, Universidade Federal de Pelotas, \\ Pelotas, RS, 96010-900, Brazil \\ ${ }^{5}$ Institute of Experimental Physics SAS, Watsonova 47, 04001 Kosice, Slovakia
}

\begin{abstract}
Using the color dipole formalism we study production of Drell-Yan (DY) pairs in proton-nucleus interactions in the kinematic region corresponding to LHC experiments. Lepton pairs produced in a hard scattering are not accompanied with any final state interactions leading to either energy loss or absorption. Consequently, dileptons may serve as more efficient and cleaner probes for the onset of nuclear effects than inclusive hadron production. We perform a systematic analysis of these effects in production of Drell-Yan pairs in $\mathrm{pPb}$ interaction at the LHC. We present predictions for the nuclear suppression as a function of the dilepton transverse momentum, rapidity and invariant mass which can be verified by the LHC measurements. We found that a strong nuclear suppression can be interpreted as an effective energy loss proportional to the initial energy universally induced by multiple initial state interactions. In addition, we study a contribution of coherent effects associated with the gluon shadowing affecting the observables predominantly at small and medium-high transverse momenta.
\end{abstract}

\section{Introduction}

The Drell-Yan (DY) process provides an important test of the Standard Model as well as a comprehensive tool for studies of strong interaction dynamics in an extended kinematical range of energies and rapidities. In this paper, we focus on dilepton pairs coming from decay of virtual $\gamma^{*} / Z^{0}$ as a probe of nuclear effects in proton-lead $(p P b)$ collisions at LHC. In this case, the DY process represents a cleaner probe than typical hadron production since the dilepton pairs have no final state interactions leading to either energy loss or absorption in the hot medium. For the same reason, no convolution with the jet fragmentation function is required and no nuclear effects are expected besides the saturation effects.

First, we give a short introduction into the color dipole picture as a framework in which the DY looks like a radiation of $\gamma^{*} / Z^{0}$ boson by a quark. We also compare calculations with the existing DY data in proton-proton $(p p)$ collisions at LHC. A more detailed recent study of DY observables for $p p$ collisions by some of the authors can be found in Ref. [1]. Then, we demonstrate that the coherence

\footnotetext{
a e-mail: michal.krelina@fjfi.cvut.cz
} 
length $l_{c}$ is large enough, and the long coherence length (LCL) limit can be safely used in calculations of the DY cross section in $p P b$ collisions.

Besides the quark shadowing which is naturally incorporated in the LCL formula, we take into account for following two important effects. The first one is the gluon shadowing which plays a greater role at LHC energies at very small fractions $x$ and can be estimated as a correction associated with the higher Fock states $\left|\gamma^{*} q G\right\rangle,\left|\gamma^{*} q 2 G\right\rangle$, etc. The second effect is the so-called effective energy loss due to the initial state interactions (ISI). The latter describes a suppression of the cross section at large dilepton $p_{T}$ which was indicated at midrapidity, $y=0$, by the PHENIX data [2] on $\pi^{0}$ production in central $d A u$ collisions and on direct photon production in central $A u A u$ collisions [3]. The same mechanism of nuclear attenuation is important, especially at forward rapidities where we expect a much stronger onset of nuclear suppression as was demonstrated by the BRAHMS and STAR data [4].

Finally, we present new results on dilepton-pion azimuthal correlation in proton-lead collisions, where the characteristic double peak structure particularly sensitive to the saturation scale is predicted.

\section{Color dipole picture}

The color dipole formalism is treated in the target rest frame where the process of DY pair production can be viewed as a radiation of gauge bosons $G^{*}=\gamma^{*}, Z^{0}$ by a projectile quark $[1,5]$. Assuming only the lowest $\left|q G^{*}\right\rangle$ Fock component the quark-nucleon differential cross section reads $[1,5,6]$

$$
\frac{d \sigma_{T, L}^{f}\left(q N \rightarrow q G^{*} X\right)}{d \ln \alpha d^{2} p_{T}}=\frac{1}{(2 \pi)^{2}} \int d^{2} \rho_{1} d^{2} \rho_{2} e^{i \vec{p}_{T} \cdot\left(\vec{\rho}_{1}-\vec{\rho}_{2}\right)} \Psi_{T, L}^{V-A}\left(\alpha, \vec{\rho}_{1}\right) \Psi_{T, L}^{V-A, *}\left(\alpha, \vec{\rho}_{2}\right) \Sigma\left(\alpha, \vec{\rho}_{1}, \vec{\rho}_{2}\right),
$$

where

$$
\Sigma\left(\alpha, \vec{\rho}_{1}, \vec{\rho}_{2}\right)=\frac{1}{2}\left(\sigma_{q \bar{q}}^{N}\left(\alpha \vec{\rho}_{1}\right)+\sigma_{q \bar{q}}^{N}\left(\alpha \vec{\rho}_{2}\right)-\sigma_{q \bar{q}}^{N}\left(\alpha\left(\vec{\rho}_{1}-\vec{\rho}_{2}\right)\right)\right)
$$

and $\vec{p}_{T}$ is the transverse momentum of the outgoing gauge boson, and $\alpha$ is a fraction of the quark LC momentum taken by the gauge boson $G^{*}$. The vector and axial-vector wave functions are decorrelated in the simplest case of an unpolarized incoming quark [5]. In this work, we take into account the presence of both interfering $G^{*}=\gamma^{*}$ and $Z^{0}$ contributions. The corresponding wave functions $\Psi_{T, L}^{V-A}(\alpha, \vec{\rho})$ can be found in Ref. [5]. The cross section for inclusive production of a virtual gauge boson in $p p$ collisions is found as follows [1]

$$
\frac{d \sigma_{L, T}\left(p p \rightarrow G^{*} X\right)}{d^{2} p_{T} d \eta d M^{2}}=J\left(\eta, p_{T}\right) \frac{x_{1}}{x_{1}+x_{2}} \sum_{f} \int_{x_{1}}^{1} \frac{d \alpha}{\alpha^{2}}\left(q_{f}\left(x_{q}, \mu^{2}\right)+\bar{q}_{f}\left(x_{q}, \mu^{2}\right)\right) \frac{d \sigma_{T, L}^{f}\left(q N \rightarrow q G^{*} X\right)}{d \ln \alpha d^{2} p_{T}}
$$

where $J\left(\eta, p_{T}\right)=\frac{2}{\sqrt{s}} \sqrt{M^{2}+p_{T}^{2}} \cosh \eta$ is the Jacobian of the transformation between the Feynman $x_{F}=x_{1}-x_{2}$ and the pseudorapidity $\eta$ variables, with Bjorken fractions $x_{1}$ and $x_{2}$. Then, $q_{f}, \bar{q}_{f}$ denote quark and antiquark PDFs, respectively, for which the CTEQ parameterisations [7] will be used, with the hard scale $\mu^{2}=p_{T}^{2}+\left(1-x_{1}\right) M^{2}$, where $M$ is a dilepton mass. In practical calculations we use several parametrisations for dipole cross sections such as GBW [8], BGBK [9] and IP-Sat [10] models.

In Figs. 1 and 2 we compare our predictions for the dilepton invariant mass distributions with recent ATLAS data in the high invariant mass range and with recent CMS data covering the $Z^{0}$ boson resonance region taking into account its interference with the $\gamma^{*}$ contribution. We can conclude that the parametrisation of the dipole cross section including the DGLAP evolution via the gluon PDF (BGBK and IP-Sat) describe the DY data better than naive GBW model as expected. 


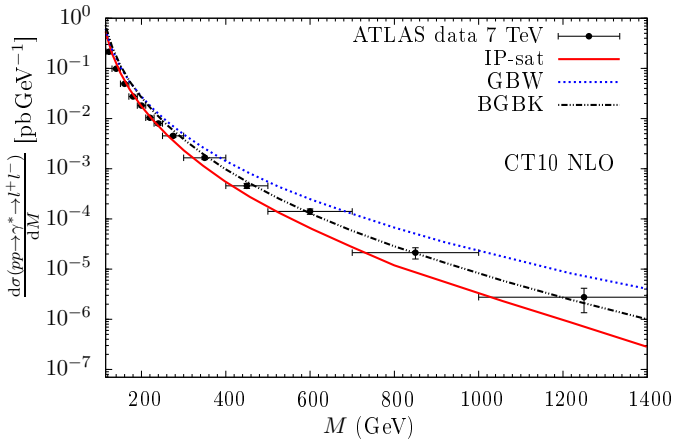

Figure 1. Predictions for the DY dilepton invariant mass distributions in $p p$ vs. data from ATLAS [11].

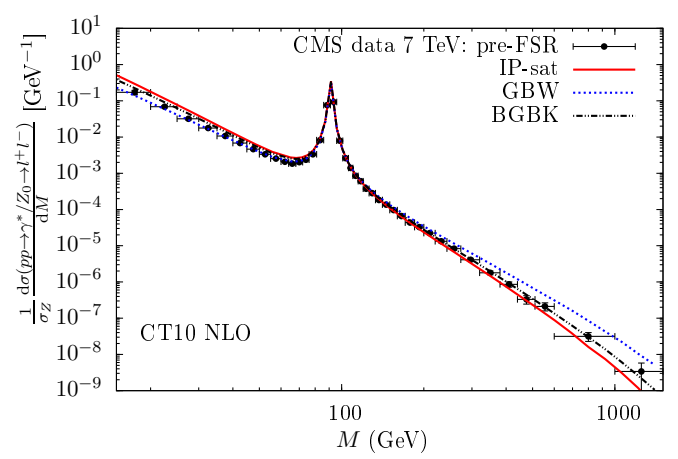

Figure 2. Predictions for DY dilepton mass distributions in $p p$ vs. data from CMS [12].

\section{Proton-nucleus interactions}

The dynamics of DY production on nuclear targets is controlled by the mean coherence length

$$
l_{c}=\frac{1}{x_{2} m_{N}} \frac{\left(M^{2}+p_{T}^{2}\right)(1-\alpha)}{\alpha(1-\alpha) M^{2}+\alpha^{2} m_{f}^{2}+p_{T}^{2}},
$$

where $M$ is the dilepton invariant mass, $m_{f}$ is the mass of a projectile quark (we take same values as in Ref. [1]) and $\alpha$ is the fraction of the light-cone momentum of the projectile quark carried out by the gauge boson. The condition for the onset of shadowing is that the coherence length exceeds the nuclear radius $R_{A}, l_{c} \gtrsim R_{A}$. In the LHC kinematic region the long coherence length (LCL) limit can be safely used in practical calculations as is demonstrated in Fig. 3. In particular, this enables us to incorporate the shadowing effects via eikonalization of $\sigma_{q \bar{q}}^{N}(\vec{\rho}, \alpha)[13]$

$$
\sigma_{q \bar{q}}^{N}(\vec{\rho}, x) \rightarrow \sigma_{q \bar{q}}^{A}(\vec{\rho}, x)=2 \int d^{2} b\left(1-e^{-\frac{1}{2} \sigma_{q \bar{q}}^{N}(\vec{\rho}, x) T_{A}(b)}\right)
$$

where $T_{A}(b)$ is the nuclear thickness function at given impact parameter $b$ normalized to the mass number $A$.

In the LCL limit, besides the lowest $\left|q G^{*}\right\rangle$ Fock state one should include also the higher Fock components containing gluons, e.g. $\left|\gamma^{*} q G\right\rangle,\left|\gamma^{*} q 2 G\right\rangle$, etc. They cause an additional suppression known as the gluon shadowing (GS). The corresponding suppression factor $R_{G}$ [15] computed as a correction to the total $\gamma^{*} A$ cross section for the longitudinal photon reads $R_{G}\left(x, Q^{2}, b\right) \approx 1-\frac{\Delta \sigma_{L}^{\left(\gamma^{*} A\right)}}{\sigma_{\text {tot }}^{\left(\gamma^{*} A\right)}}$, was included in calculations replacing $\sigma_{q \bar{q}}^{N}(\vec{\rho}, x) \rightarrow \sigma_{q \bar{q}}^{N}(\vec{\rho}, x) R_{G}\left(x, Q^{2}, b\right)$.

\subsection{Effective energy loss}

The initial-state energy loss (due to ISI effects) is expected to suppress noticeably the nuclear cross section when reaching the kinematical limits, $x_{L}=\frac{2 p_{L}}{\sqrt{s}} \rightarrow 1$ and $x_{T}=\frac{2 p_{T}}{\sqrt{s}} \rightarrow 1$. Correspondingly, a proper variable which controls this effect is $\xi=\sqrt{x_{L}^{2}+x_{T}^{2}}$. The magnitude of suppression was evaluated in Ref. [16]. It was found within the Glauber approximation that each interaction in the nucleus 


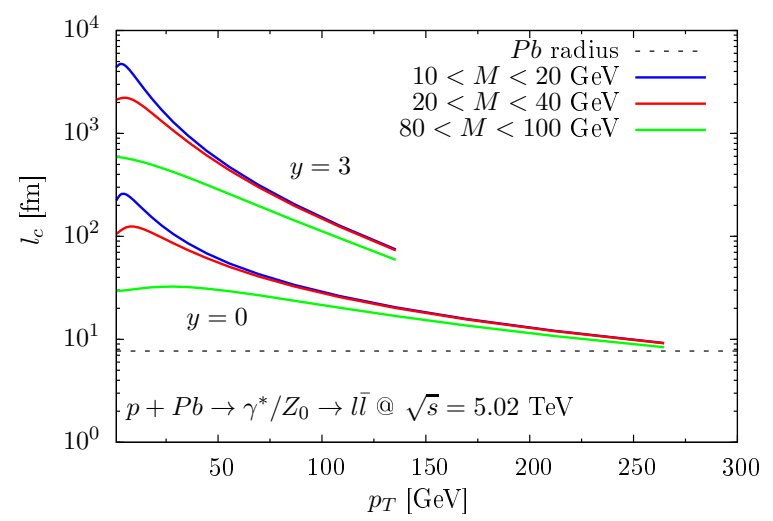

Figure 3. The mean coherence length for dilepton rapidities $y=0,3$ for different dilepton invariant mass intervals.

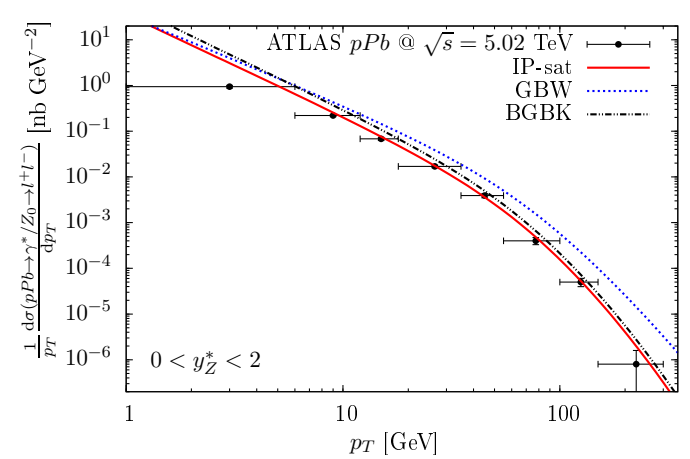

Figure 4. The dilepton $p_{T}$ distribution in $p P b$ collisions vs. ATLAS data [14].

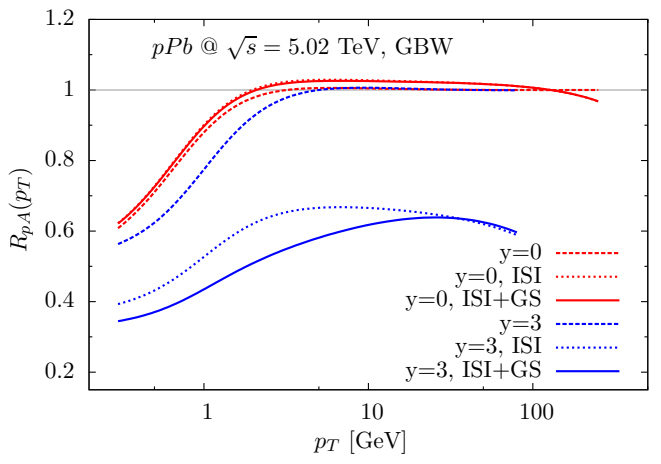

Figure 5. Predictions for the nuclear modification factor $R_{p P b}$.

leads to a suppression factor $S(\xi) \approx 1-\xi$. Summing up over the multiple initial state interactions in a $p A$ collision at impact parameter $b$, one arrives at a nuclear ISI-modified PDF

$$
q_{f}\left(x, Q^{2}\right) \Rightarrow q_{f}^{A}\left(x, Q^{2}, b\right)=C_{v} q_{f}\left(x, Q^{2}\right) \frac{e^{-\xi \sigma_{e f f} T_{A}(b)}-e^{-\sigma_{e f f} T_{A}(b)}}{(1-\xi)\left(1-e^{-\sigma_{e f f} T_{A}(b)}\right)} .
$$

Here, $\sigma_{e f f}=20 \mathrm{mb}$ is the effective hadronic cross section controlling the multiple interactions. The normalisation factor $C_{v}$ is fixed by the Gottfried sum rule (for more details, see Ref. [16]). It was found that such an additional nuclear suppression emerging due to the ISI effects represents an energy independent feature common for all known reactions experimentally studied so far, with any leading particle (hadrons, Drell-Yan dileptons, charmonium, etc).

Fig. 4 demonstrates a good agreement of our calculations for DY production in $p P b$ collisions with the data from ATLAS experiment [14]. Fig. 5 shows predictions for the nuclear modification factor $R_{p P b}$ as a function of $p_{T}$ for production of DY pairs at distinct rapidities $y=0,3$ and dilepton invariant masses in the interval $66<M<122 \mathrm{GeV}$ typical for the ATLAS measurements. The GS 
and ISI effects are irrelevant at $y=0$. However, we expect a strong nuclear suppression in the forward region $y=3$ and large $p_{T}$ 's caused by the ISI effects. Here, the GS effects give a small contribution to the nuclear suppression at $p_{T}<15 \div 20 \mathrm{GeV}$.

\section{Drell-Yan-Hadron correlations}

The correlation function $C(\Delta \phi)$ depends on the azimuthal angle difference $\Delta \phi$ between the trigger and associate particles. The azimuthal correlations are investigated through a coincidence probability defined in terms of a trigger particle which could be either the gauge boson (dilepton) or the hadron. If we assume the former as a trigger particle then the correlation function is written as [1]

$$
C(\Delta \phi)=\frac{2 \pi \int_{p_{T}^{G^{*}}, p_{T}^{h}>p_{T}^{c u t}} d p_{T}^{G^{*}} p_{T}^{G^{*}} d p_{T}^{h} p_{T}^{h} \frac{d \sigma\left(p p \rightarrow h G^{*} X\right)}{d^{2} p_{T}^{h} d \eta^{h} d^{2} p_{T}^{G^{*}} d \eta^{G^{*}} d^{2} b}}{\int_{p_{T}^{G^{*}}>p_{T}^{c u t}} d p_{T}^{G^{*}} p_{T}^{G^{*}} \frac{d \sigma\left(p p \rightarrow G^{*} X\right)}{d^{2} p_{T}^{G^{*}} d \eta^{G^{*}} d^{2} b}}
$$

where $\Delta \phi$ is the angle between the gauge boson and the hadron. The differential cross sections for $G^{*}$ and $G^{*} h$ production in momentum representation can be found in Ref. [1]. Fig. 6 demonstrates that a double peak structure emerges around $\Delta \phi=\pi$ in $p p$ collisions considering that the photon and the pion are produced at forward rapidities, close to the limit of the phase space. Taking into account the nuclear dependence of the saturation scale in the GBW model in the LCL limit we calculated the correlation functions for proton-lead collisions as is shown in Fig. 7, where we expect again the characteristic double peak structure at forward rapidity $y=4$. These results are in agreement with Ref. [17].

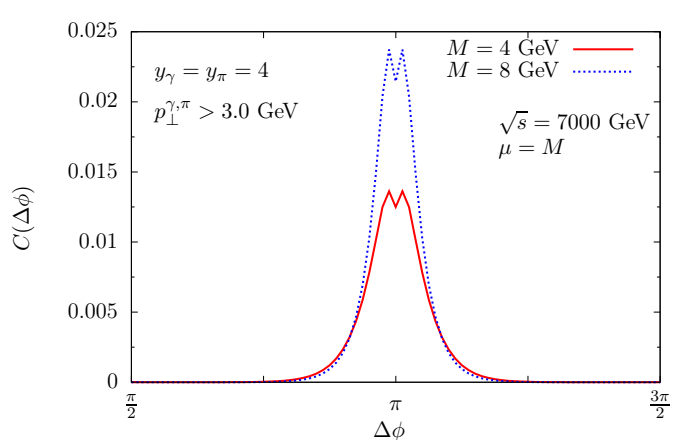

Figure 6. The correlation function for the DY-pion production in $p p$ collisions.

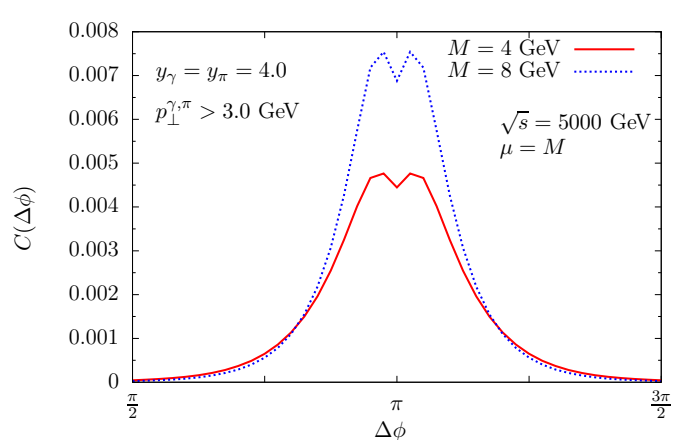

Figure 7. The correlation function for the DY-pion production in $\mathrm{pPb}$ collisions.

\section{Conclusions}

Within the color dipole picture we analyzed the DY pair production process accouning for virtual $\gamma^{*}$ and $Z^{0}$ contributions. In the case of $p p$ collisions, we found a good agreement of the differential cross section as a function of the dilepton invariant mass $M$ with the ATLAS and CMS data. We demonstrate that for production of DY pairs on nuclear targets, the LCL limit can be safely employed in the LHC kinematic region. Our calculations of differential cross section for DY production in $p P b$ collisions were compared to the ATLAS data and a good agreement has been found. We showed 
that the main source of a strong nuclear suppression, especially at forward rapidities expected at the LHC, comes mainly from the ISI corrections. A small onset of the GS is visible only at large $y=3$ for $p_{T}<15 \div 20 \mathrm{GeV}$. Investigating the anglular correlation function corresponding to associated dilepton and pion production we found a characteristic double peak structure around $\Delta \phi=\pi$ not only for $p p$ but also for $p P b$ collisions.

\section{Acknowledgements}

E. B. is supported by CAPES and CNPq (Brazil), contract numbers 2362/13-9 and 150674/20155. V. P. G. has been supported by CNPq, CAPES and FAPERGS, Brazil. R. P. is supported by the Swedish Research Council, contract number 621-2013-428. J. N. is partially supported by the grant 13-20841S of the Czech Science Foundation (GAČR), by the Grant MŠMT LG13031, by the Grant MŠMT LG15001, by the Slovak Research and Development Agency APVV-0050-11 and by the Slovak Funding Agency, Grant 2/0020/14.

\section{References}

[1] E. Basso, V. P. Goncalves, J. Nemchik, R. Pasechnik, M. Sumbera, arXiv:1510.00650 [hep-ph].

[2] S. S. Adler et al. [PHENIX Collab.], Phys. Rev. Lett. 98, 172302 (2007);

Phys. Rev. Lett. 109, 152302 (2012).

[3] S. Afanasiev et al. [PHENIX Collab.], Phys. Rev. Lett. 109, 152302 (2012);

T. Sakaguchi, Nucl. Phys. A805, 355 (2008).

[4] I. Arsene et al. [BRAHMS Collab.], Phys. Rev. Lett. 93, 242303 (2004);

J. Adams et al. [STAR Collab.], Phys. Rev. Lett. 97, 152302 (2006).

[5] R. Pasechnik, B. Z. Kopeliovich, I. Potashnikova, Phys. Rev. D86, 114039 (2012).

[6] B. Z. Kopeliovich, A. V. Tarasov, A. Schafer, Phys. Rev. C59, 1609-1619 (1999).

[7] H. Lai, M. Guzzi, J. Huston, Z. Li, P. M. Nadolsky, J. Pumplin, C. P. Yuan, Phys. Rev. D82, 074024 (2010).

[8] K. Golec-Biernat, M. Wüsthoff, Phys. Rev. D59, 014017 (1998).

[9] J. Bartels, K. Golec-Biernat, H. Kowalski, Phys. Rev. D66, 014001 (2002).

[10] H. Kowalski, L. Motyka, G. Watt, Phys. Rev. D74, 074016 (2006).

[11] G. Aad et al. [ATLAS Collab.], Phys. Lett. B725, 223 (2013).

[12] V. Khachatryan et al. [CMS Collab.], Eur. Phys. J. 75, 147 (2015).

[13] B. Z. Kopeliovich, L. I. Lapidus, A. B. Zamolodchikov, JETP Lett. 33, 595-597 (1981).

[14] G. Aad et al. [ATLAS Collab.], Phys. Rev. C92, 044915 (2015).

[15] B. Z. Kopeliovich, J. Nemchik, A. Schafer, Phys. Rev. C65, 035201 (2002).

[16] B. Z. Kopeliovich, J. Nemchik, I. K. Potashnikova, I. Schmidt, Int. J. Mod. Phys. E23, 1430006 (2014).

[17] A. Stasto, B. W. Xiao and F. Yuan, Phys. Lett. B716, 430 (2012). 